\title{
THE FAILURE OF NOTCHED COMPOSITE LAMINATES UNDER COMPRESSION USING INTEGRATED MACRO- MICROMECHANICS MODEL
}

\author{
Junghyun Ahn ${ }^{1}$ and Anthony M Waas ${ }^{2}$ \\ Composite Structures Laboratory \\ Department of Aerospace Engineering, \\ University of Michigan, Ann Arbor, MI 48109-2140
}

A combined micromechanics-macromechanics analysis methodology is developed to predict damage initiation in compressively loaded symmetric notched multidirectional laminates under remote uniaxial loading. A novel hybrid local-global finite element method in conjunction with the finite element commercial code ABAQUS ${ }^{\circledR}$ is used to evaluate the governing system of equations. The results obtained for the predictions are compared against a set of experimental results for cross-ply laminates. A failure mechanism based unified model that captures damage initiation through fiber kink banding and which accounts for stress gradient effects is presented.

\section{Introduction}

Laminated fiber reinforced polymer matrix composites (PMCs) are finding increased use in a broad range of aerospace industrial applications. More importantly, PMCs are increasingly being used as primary load bearing components not just as weight saving secondary structures. These applications include one-piece fully co-cured wing structures, gas turbine composite fan blades, and fuselage reinforcement structures.

Composite materials are superior to conventional monolithic materials for a number of reasons, noteworthy amongst these being their mechanical properties. Ashby and Jones [(1994) $]^{1}$ have formulated performance indices that can be used to select a material for a given application. PMC appears to be the material of choice for several applications. Topics such as damage tolerance, durability, low/high cycle/high fatigue under multiaxial loads, thermal cycle response and assembly joint technologies are currently being researched with a view to expanding the confidence levels associated with PMC applications.

Experience with previous applications of PMCs for rotor blades in the helicopter industry, pressure vessels, and other similar situations that call for superior tensile stiffness and tensile fatigue life have shown the superior performance of PMCs in a tensile environment. In contrast, compressive strength of PMCs is known to be less attractive [Mathews and Rawlings, (1994)]2 [Waas and Schultheisz, (1995)] ${ }^{3}$.

The response of composite laminates when subjected to mechanical loads is influenced by the material type (fiber and matrix) and configuration (stacking sequence, lay-up). In addition to these factors, geometrical parameters (cut-out, notch, thickness change, etc.) and loading characteristics (multiaxial, thermal, cyclic loading, etc) also affect the overall performance of composite laminates. One of the challenging tasks in the analysis and design with PMC based structural laminates is to improve predictive capability, particularly the development of suitable analyses tools that are robust enough to

\footnotetext{
${ }^{1}$ Researcher, Aerospace Engineering, University of Michigan, Ann Arbor, Copyright ${ }^{\circledR} 2005$ by Junghyun Ahn, published by the American Institute of Aeronautics and Astronautics, Inc; with permission

${ }^{2}$ Professor of Aerospace Engineering, Associate Fellow, AIAA.
} 
incorporate realistic geometric imperfections and manufacturing process related material imperfections (which we refer broadly to as the imperfection signature) and predict strength and damage initiation load levels. This requires that carefully conducted experiments be carried out so that the results can be used to guide the development of physics based failure models that can be incorporated in finite element based numerical codes. The present paper extends the authors' previous work [Ahn and Waas, (2002) $]^{5}$ on failure prediction in notched laminates. In particular, a micromechanics based model that consists of a region modeled discretely as layers of fibers and matrix material (the micro-region) is coupled to a region that is modeled as a homogeneous orthotropic elastic continuum. Damage is absent in the latter region, which is remote from the micro-region.

The development of the model relies on previous experimental work reported by the authors [Ahn and Waas, (2002) $]^{5}$. The procedure established using this model approach is found to provide an improved capability for the prediction of notched strength in multidirectional laminates.

\section{Modeling of Compressive Response of a Notched Multidirectional Laminate}

As shown in a companion paper [Ahn and Waas, (2002)], when a multidirectional laminate containing a circular hole is subjected to remote compressive loads, failure in the form of a microstructural instability is initiated in the vicinity of the hole. For cross-ply laminates, the failure is triggered by zero-degree ply instability, characterized by fiber kinking. For the case of quasi-isotropic laminates, which consist of zero and 45-degree plies, two types of failure mechanisms compete against each other. These are failure initiation due to fiber instability in the zero plies and failure initiated by matrix shear response, responsible for interface (fiber/matrix) cracking and/or shear failure in the 45 degree plies. In the present paper, only cross-ply laminates are considered. The dominant failure mode for this type of laminates has been reported as zero ply kinking failure [Ahn and Waas (1999)] ${ }^{6}$, [Soutis et al. (1991)] ${ }^{12}$.

The micromechanics based modeling approach used in previous papers, [Ahn and Waas (1999) $]^{6}$, [Ahn and Waas, (2004)] ${ }^{15}$, employed several simplifying assumptions aimed at reducing the "size" of the numerical problem. In particular, symmetry considerations were used to mesh a region that is half of the micromodel region used in the present study, in effect eliminating any non-symmetric instability modes. The overall effect of this assumption is to introduce additional stiffening, thus generating larger than measured failure initiation loads.

As an intermediate step in the evolutionary process of developing a unified model that spans several length scales, the microregion was extended (referred to as the full model) to either side of the hole as shown in Figure 1 and Figure 2. A schematic of the notched cross-ply laminates are shown in Figure 1. In Figure 1 and Figure 2, the region $A B C D$ shows the boundary of the meshed area that represents the microregion within which the fibers and matrix are modeled as discrete layers. This micro-region was decoupled from the remote areas through an analytical solution that was used to compute the remote displacement field. These displacement fields were used to "load" the micro region, in order to study its stability characteristics. The results of the full model were presented in a previous paper [Ahn and Waas, AIAA-2004-1844] $]^{15}$.

A fully extended model (microregion embedded within a homogenized orthotropic lamina, which we refer to as the extended model) is shown in Figure 3 and is the subject of this paper. In this extended model, the microregion is modeled as a continuum within which the fibers and matrix are modeled as discrete layers. The region immediately outside the microregion is modeled as a homogenized orthotropic lamina. The Lekhnitskii orthotropic elasticity [Lekhnitskii, (1968)] ${ }^{6}$ solution for a homogenous infinite plate containing a centrally placed cutout and subjected to remote uniaxial loading is used to compute the displacement fields along the edges EE1-F1F, FG, GH and HE (Figure 3) of the microregion. 
Because the entire laminate is modeled according to classical lamination theory in the areas remote from the hole, the Lekhnitskii solution provides the required strain fields from which the displacement fields along the boundaries EE1-F1F, FG, GH and HE (Figure 3) of the microregion are computed. These displacement fields correspond to a unit far-field uniaxial compressive load. For other multi-axial loading proportional loading situations, a similar procedure can be adopted. For the sake of brevity, the uniaxial loading case is used to present the methodology and demonstrate its usefulness.

The failure initiation analysis based on micromechanics is carried out in a manner similar to that described in [Ahn and Waas, (1999)] ${ }^{6}$. For the FEA analysis, a rectangular mesh containing 16,006 elements (parabolic plane strain) and 48,533 nodes, with two degrees of freedom ( $u$ and v) per node was chosen within the microregion. Convergence of the solutions with respect to microregion size and microregion mesh density were both checked by incrementally increasing the microregion size and mesh density until no substantial change in the salient features (and values) associated with the response resulted. These features included examination of the in-plane stress and displacement fields.

A flow chart of the analysis procedure is indicated in Figure 4(a). First, an elastic eigenvalue analysis of an isolated (decoupled from the rest of the plate) microregion model is carried out in order to obtain in-plane eigenmodes that correspond to the entire plate having the same global load corresponding to the subsequent response analysis that follows. The eigenmode shape associated with the smallest non-zero eigenvalue is used to perturb the FE mesh corresponding to the microregion. Because the eigenmode shapes are localized, the outer boundaries of the microregion (ABCD) are unperturbed and they smoothly attach to the outer homogenized orthotropic region of the laminate. That is, the interface between the microregion (ABCD) and the outer homogeneous (orthotropic) region is modeled as 'glued' contact interface, to avoid excessive mesh transition by assuming the interface as a contact interface. Response analyses are carried out using the arc length method option provided in ABAQUS ${ }^{\circledR}$. During the response analysis, the boundaries EE1-F1F, FG, GH, and HE are subjected to displacement fields that are computed from the orthotropic elasticity solution. The traction load, previously applied along the edge AB in the full model (see [Ahn and Waas, (2004)] ${ }^{15}$ ), is no longer necessary because the current model boundary is extended to the free edge of the hole. Simulations were carried out for a series of imperfection magnitudes for a hole size $(0.25 \mathrm{R})$.

The eigenmodes provide the perturbation shape for the initial fiber misalignment but not the absolute magnitude of perturbation. Thus, the user must specify the imperfection magnitude. In the present work, this is achieved as follows; as shown in Figure 4(b), the maximum amplitude, $\delta$, of the lowest eigenmode (which occurs at the free notch edge - indicated as point J in figure 2) is chosen such that the fiber misalignment angle $\phi$ can be controlled. Since the characteristic half wave length $\lambda$, of the localized eigenmode shape is known, $\delta$ is chosen such that $\phi$ assumes the intended value. Several simulations corresponding to different values of $\phi$ were completed. In each case, the maximum load associated with the global load proportionality factor applied in proportion to the initial displacement field is computed. In a typical run, the relation between the applied load and the axial displacement (average axial displacement of the microregion along the edge BC) is as shown in Figure 4(a) - inset "response of microregion". The load rises almost linearly, followed by a well-defined peak load and subsequent snap back in the response. The peak load corresponds to the onset of instability while the snap back part of the response corresponds to the formation of a kink band (in which the deformation is localized) with simultaneous growth and rotation of fibers within the kink band. The far-field applied loads corresponding to the peak loads can be plotted as a function of imperfection magnitude (see Figure 4(a) inset "extrapolation to perfect case"), from which, by extrapolation, the maximum applied remote stress corresponding to perfectly straight fibers can be extracted. Thus, the process outlined not only gives the maximum strength of the laminate corresponding to the given (or measured) fiber misalignment, but also 
provides the maximum attainable strength of the laminate. The latter could be used as an upper bound on strength, while the strength corresponding to a fiber misalignment of $1.5^{\circ}$ (say) could be used as the lower bound. The amount of fiber misalignment is dependent on the manufacturing imperfection signature. That is, different manufacturing processes give rise to different statistics of initial misalignment. Once the imperfection signature is known, then the values of $\phi$ that are associated with a specific manufacturing process can be extracted during the inspection (using techniques such as ultrasonic methods, x-ray techniques etc.) phase of hardware. For pre-preg based laminates nominal values in the range of $\phi=0.5^{0}-2^{0}$ is reasonable.

Throughout the analyses, the fiber (IM7) is assumed to be linearly elastic (see Table 1), and the matrix (977-3 toughened epoxy) property within the laminate (in-situ) is evaluated from a $+45 /-45$ laminated coupon test. This test is described in the next section. Thus, the in-situ nonlinear stress-strain response of the matrix behavior is incorporated in the present analysis. The matrix is modeled as a J2 incremental flow theory solid with isotropic hardening, and this is also verified through measurement.

\section{In-Situ Matrix Characterization}

To evaluate the material properties of the matrix within the laminate (in-situ matrix properties), $\mathrm{a}( \pm 45)_{n s}$ coupon test (Figure 5$)$ at room temperature was performed following the procedure of ASTMD3518-76 [ASTM Standard, (1982)] ${ }^{8}$. The elastic properties of the lamina and the complete shear stressshear strain behavior of an IM7/977-3 in the principal material coordinate system were obtained from this test. The procedure used to generate the data consists of subjecting a $( \pm 45)_{n s}$ angle ply laminate to uniaxial compression and measuring the laminate strains $\varepsilon_{x x}$ and $\varepsilon_{y y}$ and the applied remote stress on the laminate. Note that, for this test, $\gamma_{x y}=\varepsilon_{x x}-\varepsilon_{y y}$. The data shown in Figure 6 from this test can be used to extract the complete nonlinear shear stress - shear strain response of the in-situ matrix (977-3) as discussed below. Begin by assuming that the 977-3 matrix material can be modeled as an elastic-plastic solid obeying the small strain $J 2$ flow theory of plasticity [Lubliner, (1998)] $]^{9}$. Then, from the elastic (linear) portion of the curve in Figure 6, the inplane lamina shear modulus $G_{12}$ is first obtained. In the lamina principal coordinates, one has,

$$
\begin{aligned}
& \varepsilon_{11}=\varepsilon_{22}=\frac{\varepsilon_{x x}+\varepsilon_{y y}}{2} \\
& \gamma_{12}=2 \varepsilon_{12}=\varepsilon_{x x}-\varepsilon_{y y}
\end{aligned}
$$

and

$$
\begin{aligned}
& \sigma_{11}=\frac{\sigma_{x x}}{2} \\
& \sigma_{22}=\frac{\sigma_{x x}}{2} \\
& \tau_{12}=\frac{\sigma_{x x}}{2}
\end{aligned}
$$

Thus, using (1) and (2) above and the definitions of equivalent stress, $\bar{\sigma}$ and equivalent plastic strain increment, $d \bar{\varepsilon}^{p}$ [Lubliner, (1998)] $]^{9}$, the data in Figure 7 can be used to construct a plot of $\bar{\sigma}$ against $\bar{\varepsilon}^{p}$.

According to the $J 2$ flow theory of plasticity with a Mises-Henky yield condition, the ratio of the increment of each plastic strain component to its corresponding deviatoric stress component remains constant [Lubliner, (1998)] ${ }^{9}$, 


$$
\frac{d \varepsilon_{i j}^{p}}{\hat{\sigma}_{i j}}=d \lambda
$$

Using (3), and the relation between $\bar{\sigma}$ and $d \bar{\varepsilon}^{p}$, the nonlinear portion of the shear stress $\left(\tau_{12}\right)$-shear strain $\left(\gamma_{12}\right)$ response curve for a single lamina can be extracted. The curve thus obtained for IM7/977-3 system is shown in Figure 8. The instantaneous slope of the curve in Figure 8 is the tangent shear modulus of the lamina, $G_{12}^{T}\left(\tau_{12}\right)$, while the ratio of shear stress to shear strain is the secant shear modulus. The approximate Halpin-Tsai relations [Daniel and Ishai, (1994)] ${ }^{10}$ are used to extract the variation of the in-situ matrix shear modulus $G_{m}^{S}\left(\tau_{12}\right)$,

$$
\begin{aligned}
& G_{m}^{S}=G_{12}^{S}\left(\frac{1+\xi_{2} \cdot \eta_{2} \cdot v_{f}}{1-\eta_{2} \cdot v_{f}}\right)^{-1} \\
& \eta_{2}=\frac{G_{12 f}-G_{m}}{G_{12 f}+\xi_{2} G_{m}}
\end{aligned}
$$

with $\eta_{2}=1$ for random packing of fibers. With $G_{m}^{S}\left(\tau_{12}\right)$ so obtained, the in-situ matrix shear stressshear strain curve is as shown in Figure 9. In a similar manner, the in-situ uniaxial stress-strain curve in compression for the matrix is also obtained (Figure 10). This uniaxial response curve was discretized and used in the input data file to represent the matrix material in the microregion for the micromechanics based finite element analysis carried out using ABAQUS®.

\section{FEA Results and Discussion}

A typical load response behavior of a microregion within a cross ply laminate model is shown in Figure 11 and a series of deformed plots of the microregion showing the initiation and propagation of damage in the form of kink banding is as shown in Figure 12, for a specimen with a hole size 0.25 inch radius, with a fiber misalignment of $\phi=0.87$.

The microregion response follows a linear path up to point(1). Although local matrix yielding (in areas of the microregion near the cutout) is indicated prior to the attainment of point(2), the total integrity of this region is not affected much from the matrix yielding because, (1) the area of yielding is small compared to the overall size of the microregion and (2) the fiber rotations are small up to the point of maximum load (point(3)). The sudden load drop is defined as the failure initiation point (the corresponding load, therefore, is the failure initiation load). As loading increases, the fibers in the areas where the matrix has become "softer" start to rotate, resulting in a drop of the resultant force. Once the load drop initiates (characterized by high localized deformation at the hole center), the band of localized deformation continues to propagate into the unkinked material until it reaches the outer micromodel boundary.

Simulations corresponding to several different fiber misalignment angles were carried out and the results for maximum load so obtained were plotted against imperfection amplitude in order to determine the upper bound strength corresponding to perfectly straight fibers (Figure 13).

Another failure mechanism observed with laminates that include a dominant number of off-axis plies was matrix shearing eventually leading to fiber/matrix interface fracture. An analysis that includes incorporation of cohesive zone models much in the spirit of [Song and Waas (1995)] ${ }^{11}$, is relegated to the future. For now, we observe the good agreement between the far-field load corresponding to the maximum load prediction and the experimentally measured failure loads for kink banding as shown in Figure 13, especially for the case of small imperfection $\left(\phi=0.45^{\circ}\right)$ and a hole size of 0.25 inch radius. The 
results in Figure 13 indicate strong sensitivity to fiber misalignment. Results such as those in Figure 13, can be generated for other hole sizes. For instance, in earlier work, the effect of hole size (i.e. notch sensitivity) was reported using the full model [AIAA-2004-1844] ${ }^{15}$. The results for notch sensitivity from the full model are reproduced here and are shown in Table 2. The results in Table 2 show that the present extended model is, as expected, less stiff than the full model. For example, with the full model, for the case of hole radius 0.25 inch, an imperfection of $\left(\phi=1.5^{0}\right)$, produces agreement with test data. With the extended model, for the same hole size, $\left(\phi=0.45^{\circ}\right)$ produces an exact match.

The trends for compressive strength as a function of hole radius as indicated in Table 2 are very similar to that reported experimentally for comparable fiber/matrix systems (for instance, see Soutis et al. $(1991)^{12}$ and Starnes and Williams (1982) ${ }^{13}$. The good agreement between prediction and experiment for the single hole size that has been examined experimentally lends confidence to the contention that failure prediction based on zero ply kinking is the governing mode of failure initiation in notched cross-ply laminates. Such a conclusion, based only on experimental results, has been reached earlier by several previous investigators-see for example, Soutis, Fleck and Smith (1991) ${ }^{12}$ and Khamseh and Waas (1997) ${ }^{14}$.

\section{Concluding Remarks}

A micromechanics-macromechanics based finite element local-global model for predicting the compressive strength of notched cross-ply composite laminates has been presented. The effect of initial fiber misalignment on the failure initiation load has been adequately captured. Ongoing studies are aimed at establishing notch sensitivity, similar to what has been reported earlier (see [Ahn and Waas, (2004) $]^{15}$ ). The very good comparison between experiment and analysis for a single notch size lends confidence to the veracity of the model. Using measured fiber and matrix nonlinear material properties, geometry and stacking of the laminate, the model has accurately predicted the notched compressive strength of laminates.

\section{References}

1. Ashby M.F. and Jones, D., “Engineering Materials 2", Pergamon, 1994

2. Mathews, F. L. and Rawlings, R., "Composite Materials: Engineering and Science", Chapman and Hall, London, 1994

3. Waas, A. M., and Schultheisz, C. R., "Compressive failure in Composites, Part II", Progress in Aerospace Science, Vol.32, pg. 43-78, 1995.

4. Starnes, J., Rhodes, M.D., and Williams, J.G., "Effect of Impact Damage and Holes on the Compressive Strength of a Graphite/Epoxy Laminate", Nondestructive Evaluation and Flaw Criticality for Composite Materials, edited by R. B. Pipes, ASTM STP 696, pg. 145-171, 1979.

5. Ahn, Junghyun and Waas, A.M., "Prediction of Compressive Failure in Laminated Composites at Room and Elevated Temperature", AIAA JOURNAL, Volume 30, Number 2, pg. 346 - 368, 2002

6. Ahn, Junghyun and Waas, A.M. "Finite Element Model for Compressive Failure of Notched Uniply Composite Laminates under Remote Biaxial Loads" ASME Journal of Engineering Materials and Technology: Vol.121, pg. $001-007,1999$

7. Lekhnitskii S., "Theory of Elasticity of an Anisotropic body", Text, Godden-Day, 1968.

8. “D 3410-75 Standard Test Method for Compressive Properties of Unidirectional or Cross-ply FiberResin Composites", 1982, Annual Book of ASTM Standards, Part 36, American Society for Testing and Materials, Philadelphia, PA, pg. 872-880., 1982 
9. Lubliner Jacob, "Plasticity Theory", Text, Prentice Hall, 1998.

10. Daniel, Isaac M. and Ori Ishai, "Engineering Mechanics of Composite Materials", Oxford University Press, New York, 1994.

11. Song, S.J. and Waas, A.M., "An Energy Method based Model for Mixed Model Failure of Laminated Composites", AIAA Journal, vol. 33, No. 4, pg. 739-745, 1995.

12. Soutis, C., Fleck, N. and Smith, F., "Failure Prediction Technique for Compression Loaded Carbon Fiber-Epoxy Laminate with an Open Hole", J. Composite Materials, 25, pg. 1476- 1498, 1991

13. Starnes, J. and Williams, J.G. (1982), Failure characteristics of Graphite/Epoxy structural components loaded in compression, NASA TM 84552.

14. Khamseh A. and Waas A., "Failure Mechanisms of Composite Plates with a Circular Hole under remote Biaxial Planar Compressive Loads", ASME J. Materials and Technology, vol. 119, pg. 56-64, 1997

15. Ahn, Junghyun and Waas, A.M., "Stress Gradient Effects on Notched Composite Failure using a Local-Global Approach", 45'th AIAA-ASME-SDM Conference AIAA-2004-1844

\section{Tables and Figures}

\begin{tabular}{|c|c|c|c|c|c|}
\hline Material & $\begin{array}{c}\mathrm{E}_{11} \\
(\mathrm{Msi})\end{array}$ & $\begin{array}{c}\mathrm{E}_{22} \\
(\mathrm{Msi})\end{array}$ & $\begin{array}{c}\mathrm{G}_{12} \\
(\mathrm{Msi})\end{array}$ & $v_{12}$ & $\begin{array}{c}\text { Thicknes } \\
\mathrm{s}(\mathrm{in})\end{array}$ \\
\hline $\begin{array}{c}\text { IM7 / 977-3 } \\
\text { Epoxy }\end{array}$ & 23.5 & 1.21 & 0.72 & 0.30 & 0.0052 \\
\hline IM7 Fiber & 42 & & & 0.25 & $\begin{array}{c}2.756 \mathrm{E}- \\
04\end{array}$ \\
\hline $\begin{array}{c}977-3 \\
\text { Epoxy }\end{array}$ & 0.7 & & & 0.34 & $\begin{array}{c}2.444 \mathrm{E}- \\
04\end{array}$ \\
\hline
\end{tabular}

Table 1 Zero ply material properties of the 48 ply graphite / 977- 3 epoxy composites

\begin{tabular}{|c|c|c|c|}
\hline Hole Radius (in) & 0.0625 & 0.125 & 0.25 \\
\hline $\begin{array}{c}\text { Max. Load (no } \\
\text { imperfection) } \\
\text { (ksi) }\end{array}$ & 163 & 123 & 75 \\
\hline $\begin{array}{c}\text { Max. Load (1.5 deg } \\
\text { imperfection) } \\
\text { (ksi) }\end{array}$ & 78 & 63 & $\begin{array}{c}44 \text { (matching } \\
\text { test result) }\end{array}$ \\
\hline
\end{tabular}

Table 2 Analyses Results using Full Model (from Ahn and Waas, 20045) 


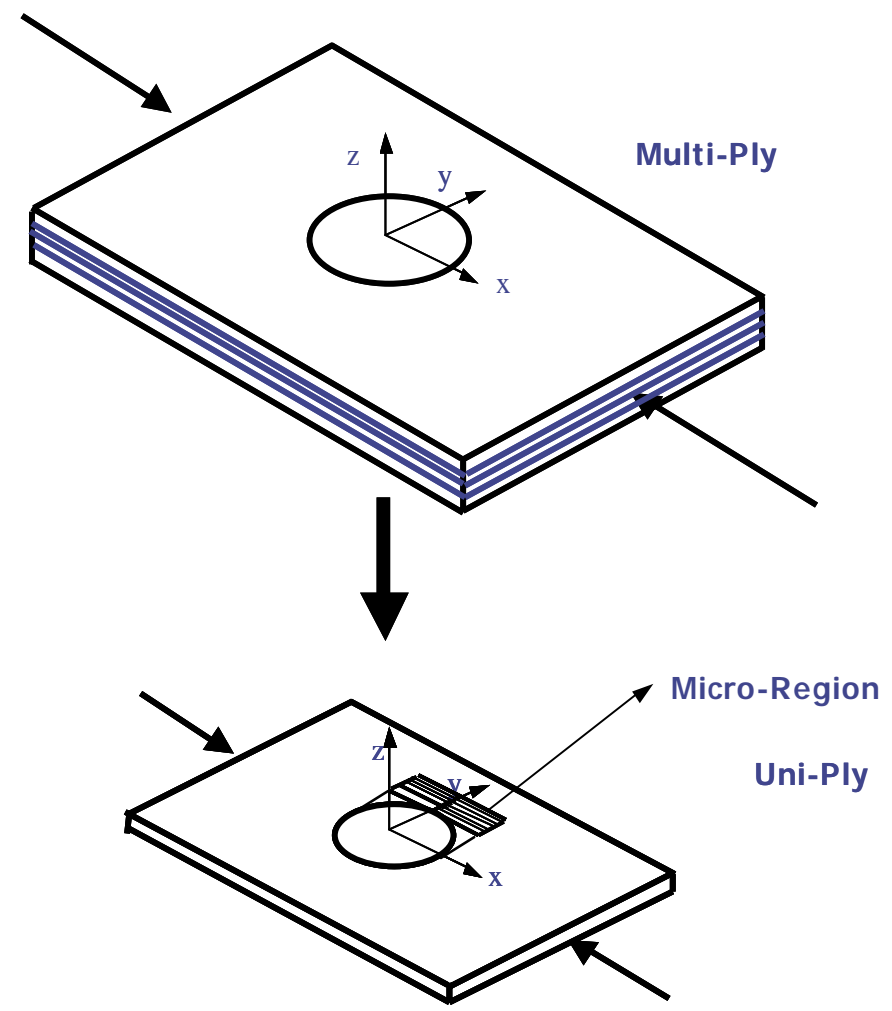

Figure 1. Problem configuration for Crossply Laminates

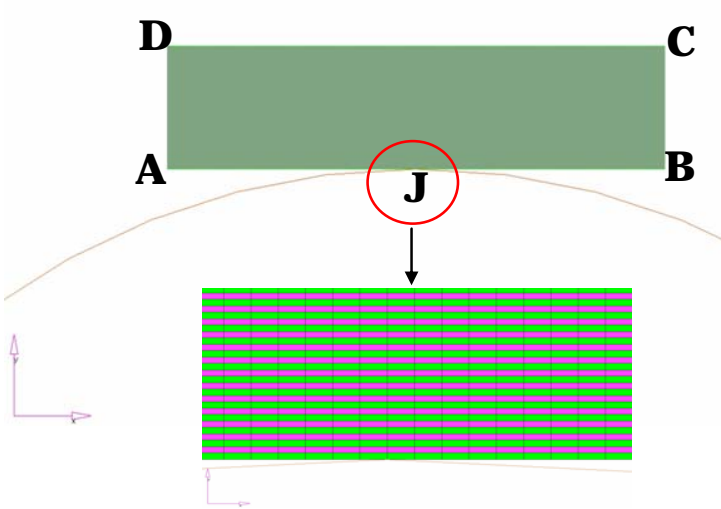

Figure 2. Full Model 
$\mathbf{H}$

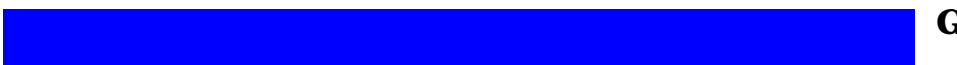

G

$\mathbf{E}$

E1
F1

$\mathbf{F}$

Figure 3. Extended Model
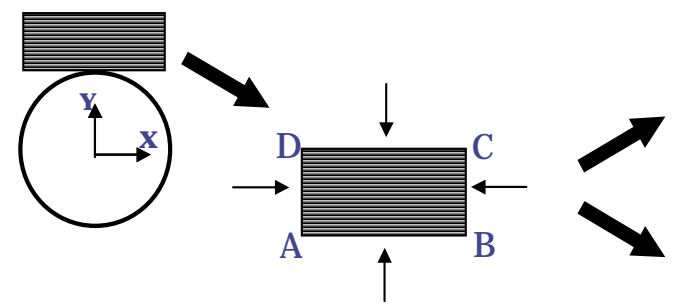

Eigenvalue Analysis (Model Perturbation)

Nonlinear Response Analysis

Static Analysis (Force Field)

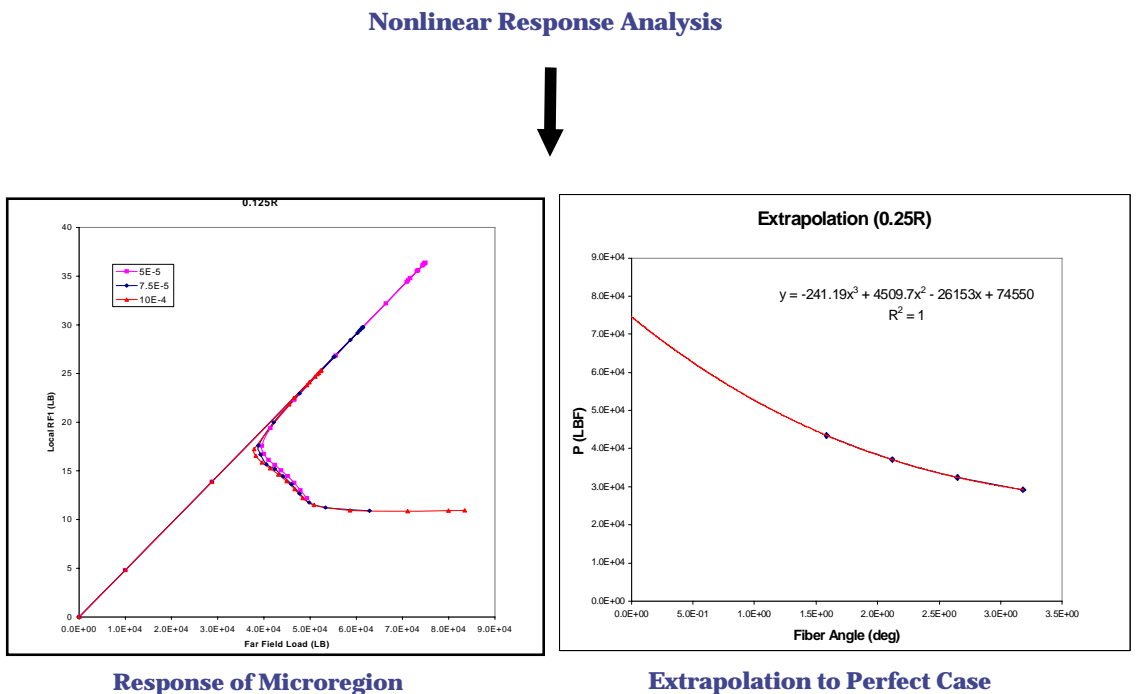

Figure 4(a) Imperfection Sensitivity Analysis Procedure 


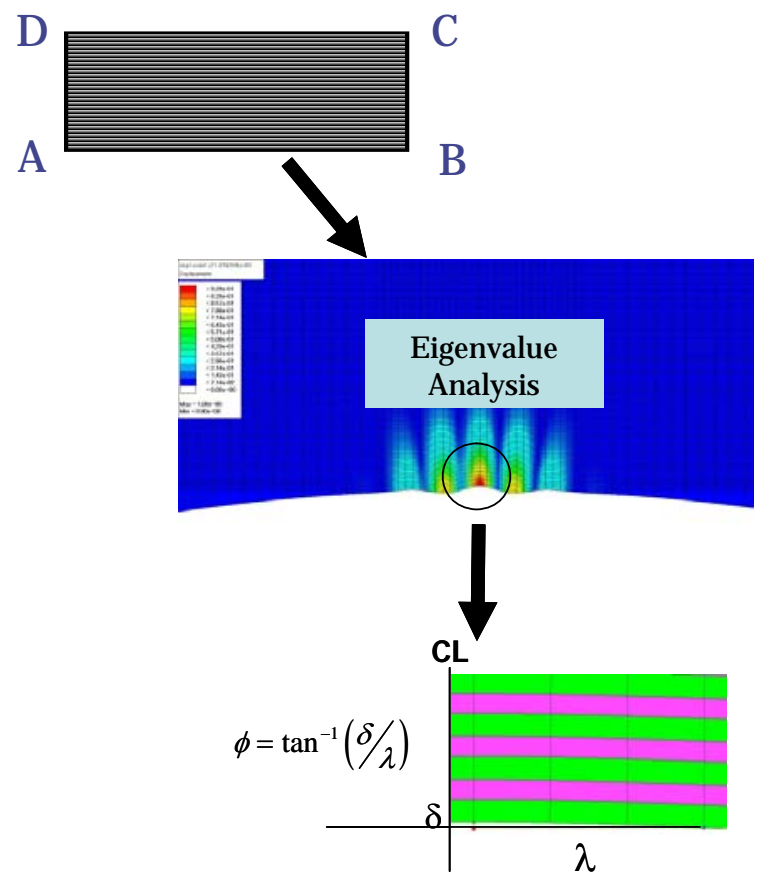

Figure 4(b) Details of initial imperfection

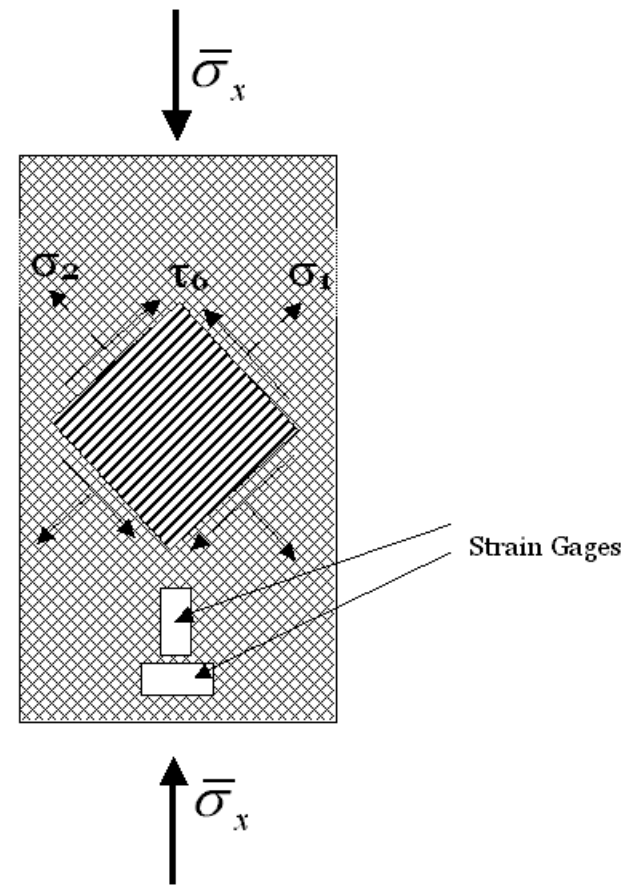

Figure 5. $+45 /-45$ coupon test of a specimen taken from the cross ply laminates 
AIAA-2005-1954

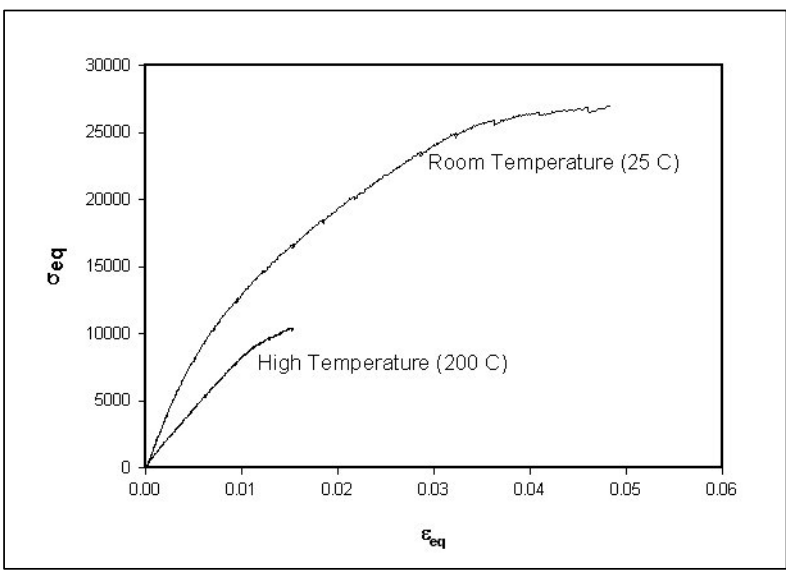

Figure 6. $+45 /-45$ compression experiment result. Equivalent stress vs. equivalent plastic strain.

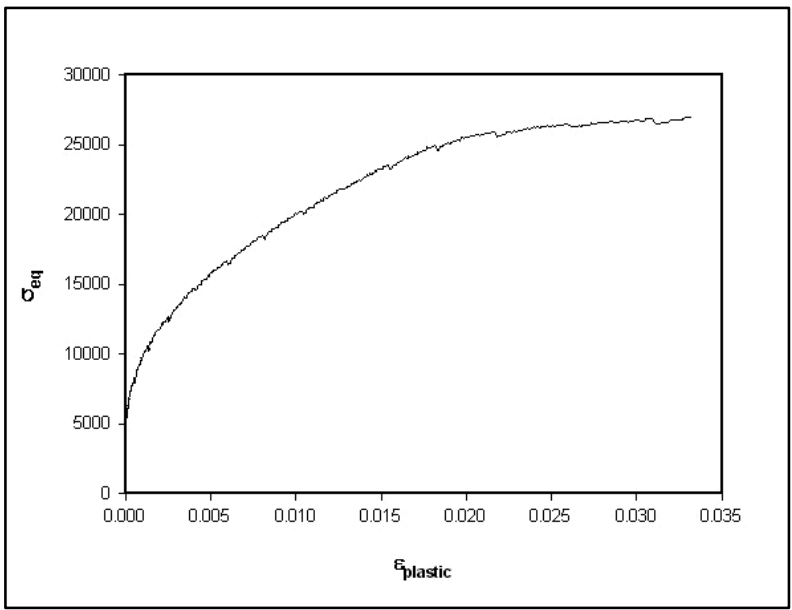

Figure 7. Equivalent stress vs. equivalent plastic strain (room temperature). 


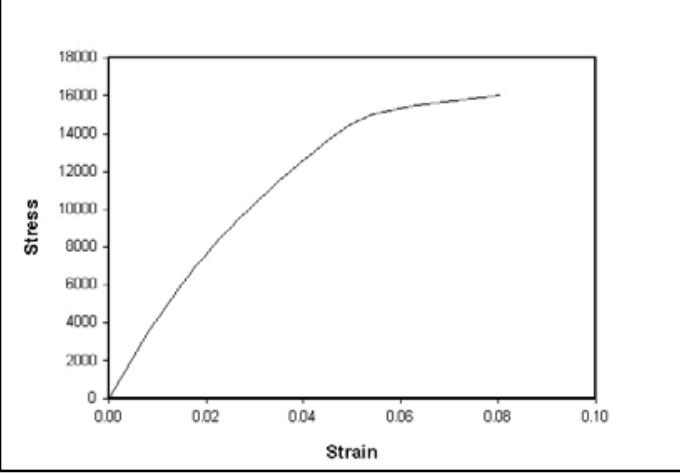

Figure 8 Shear Stress, $\tau_{12}$ vs. Shear Strain, $\gamma_{12}$ curve for IM7 I 977-3 based on J2 Flow Theory of Plasticity and the data in Figure 7.

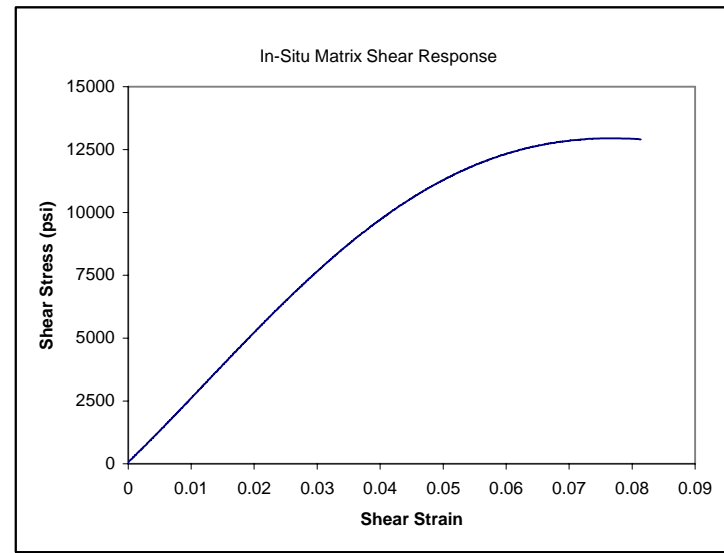

Figure 9. The in-situ Shear Stress vs. Shear Strain response of the matrix.

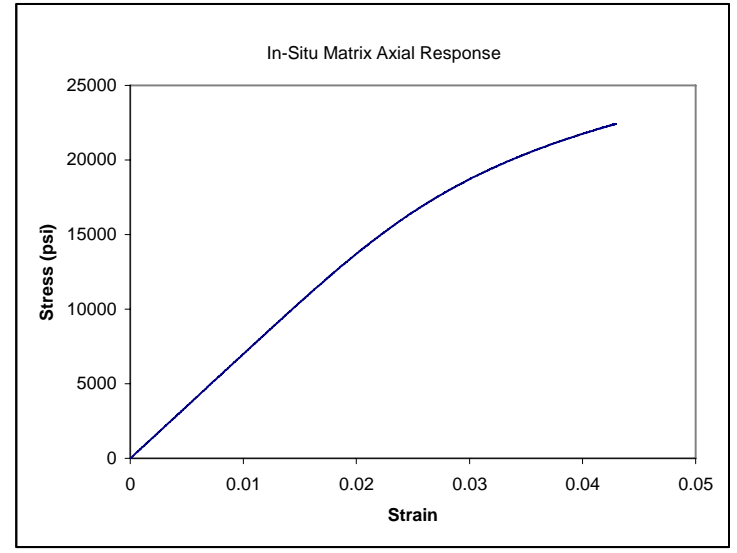

Figure 10. The in-situ uniaxial stress vs. uniaxial strain response of the matrix. 


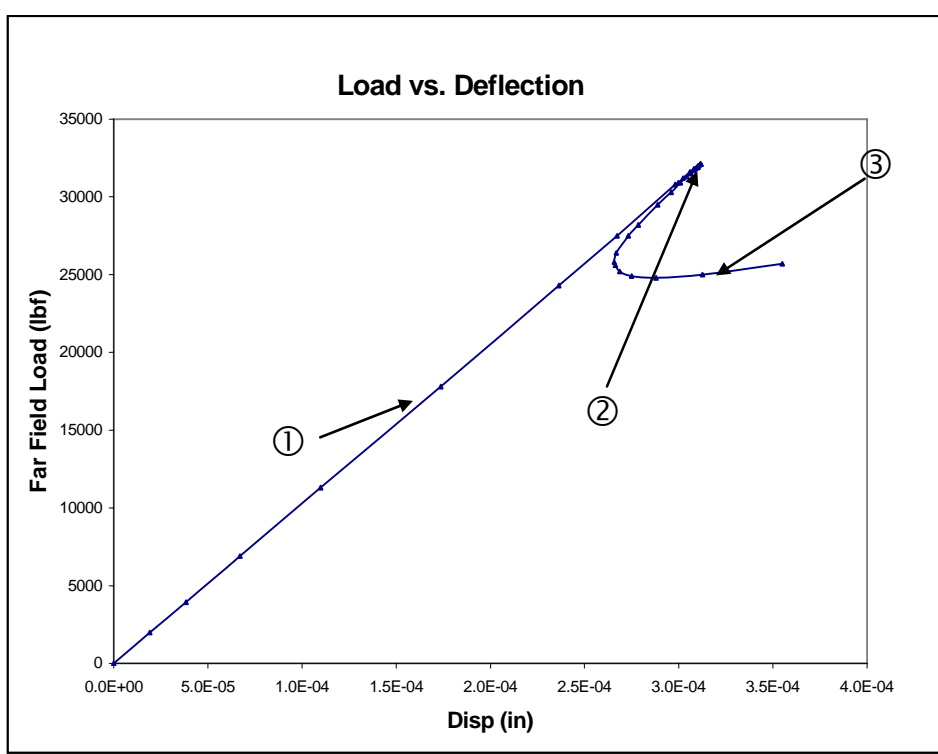

AIAA-2005-1954

Figure 11. Typical Response Curve of the microregion (0.25R)
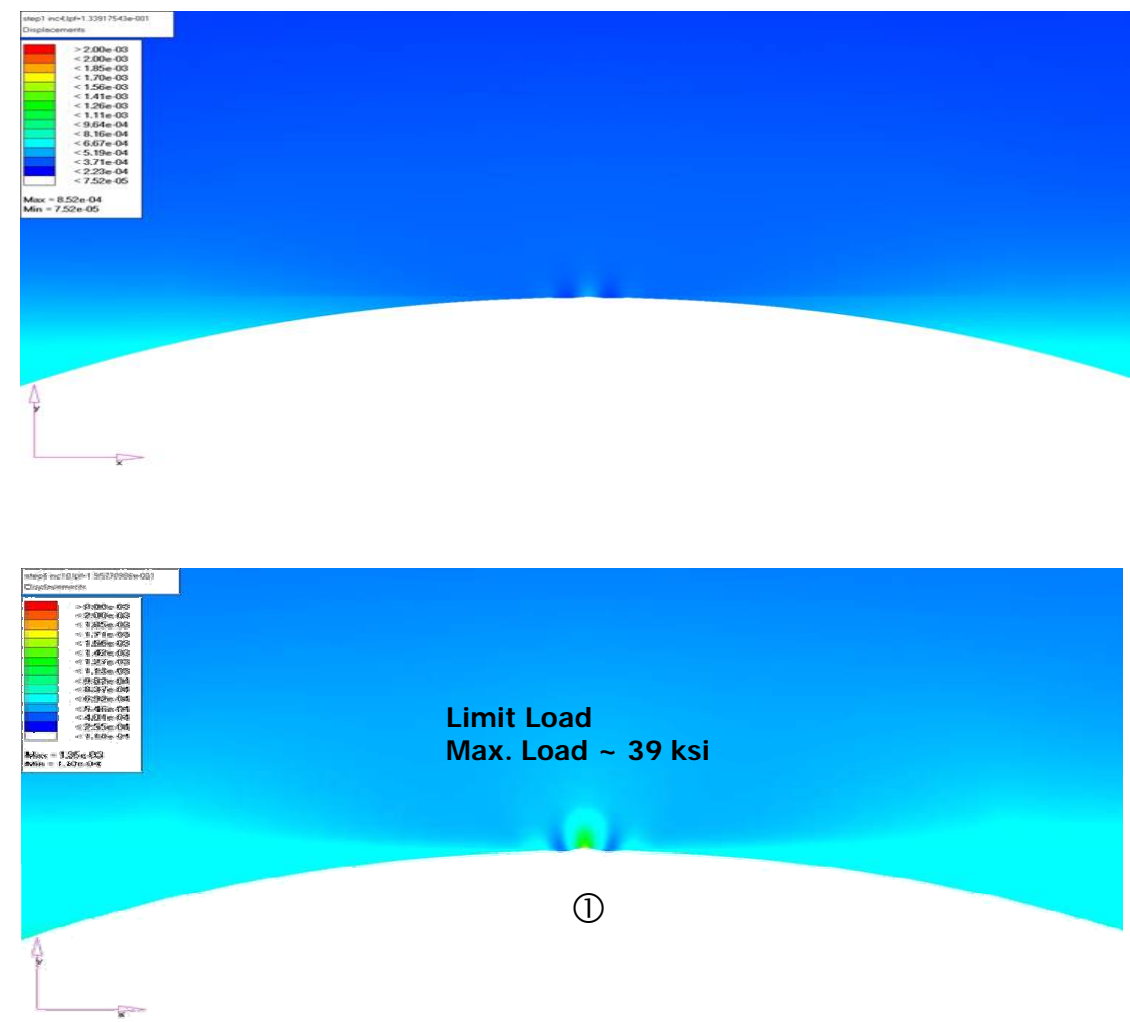


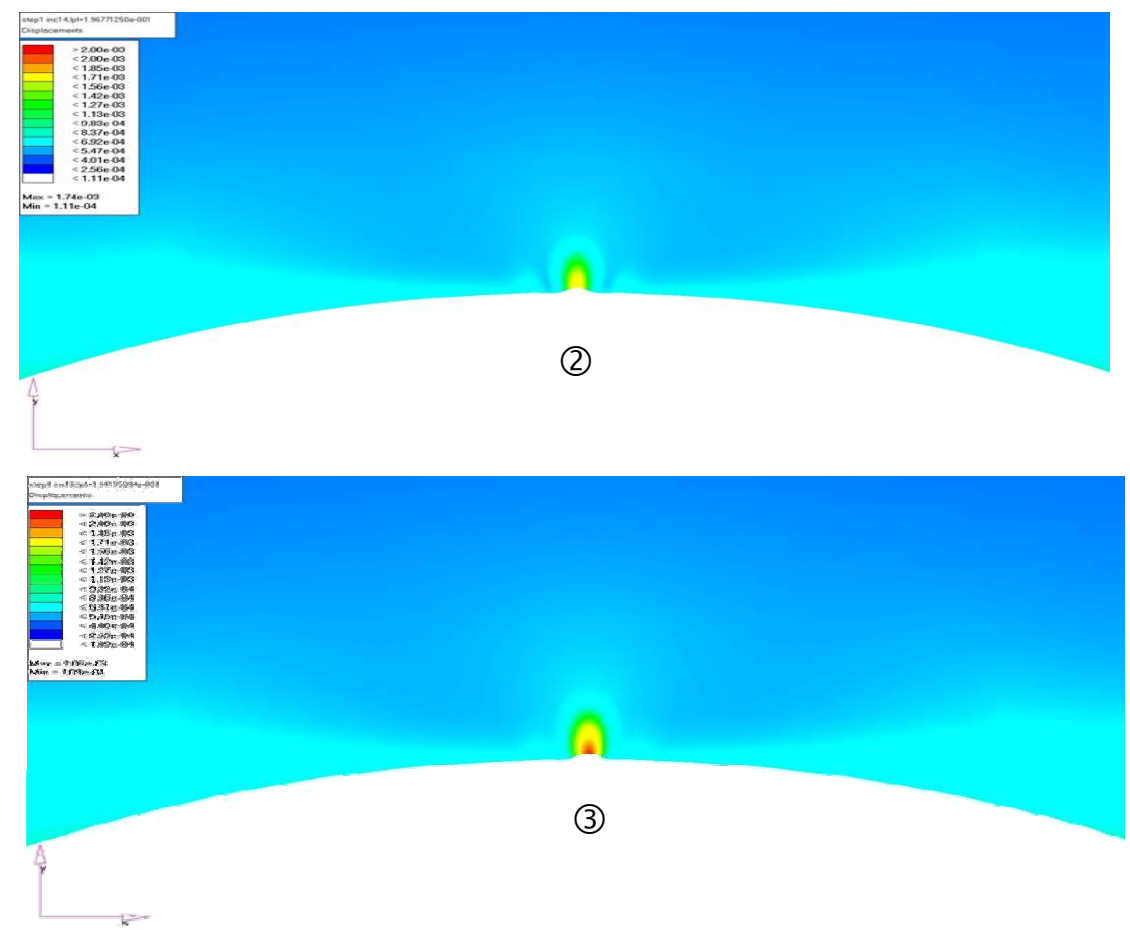

Figure 12. Contour plot of Displacement (equivalent) in the Microregion

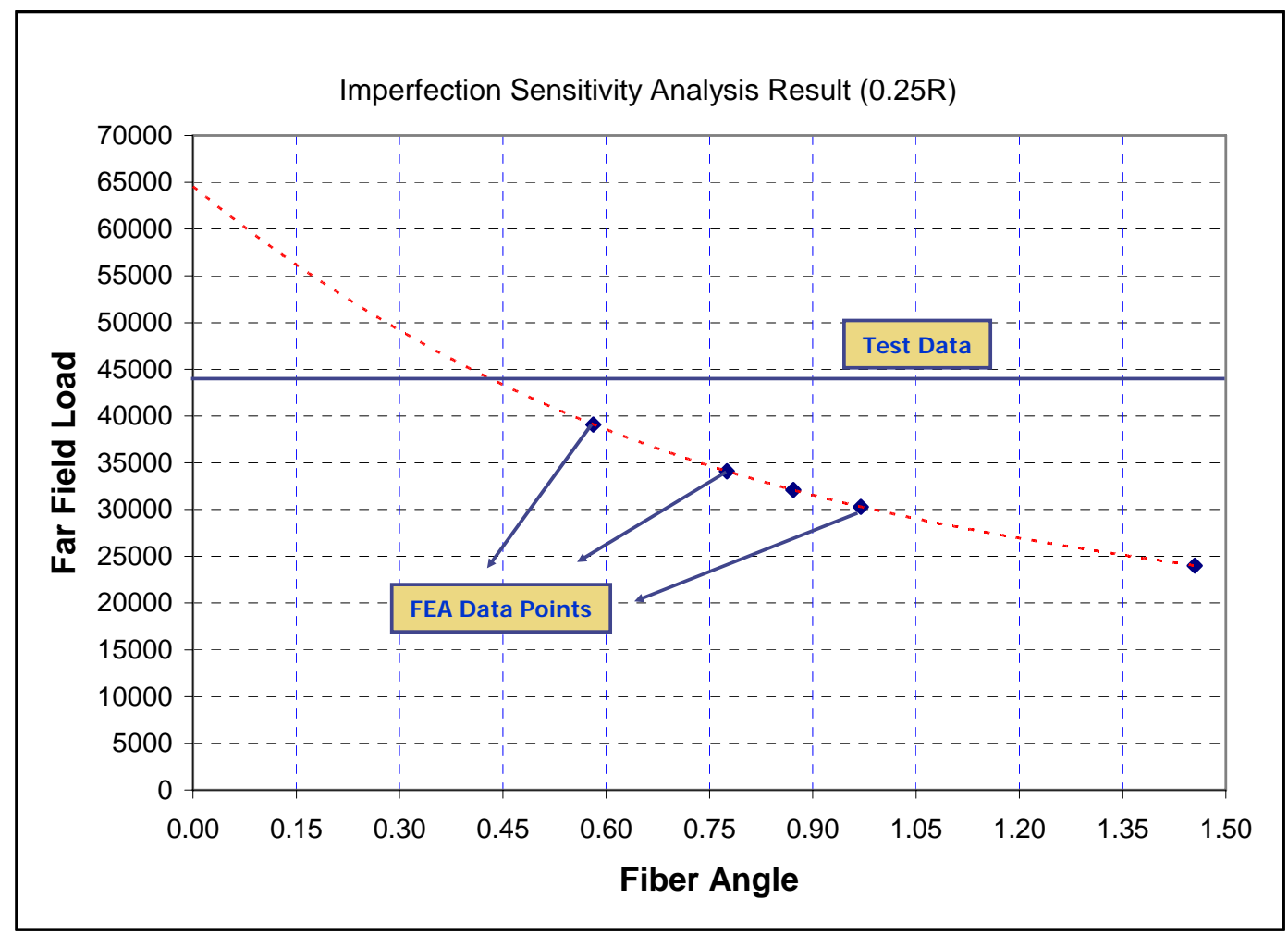

Figure 13. Analysis Result for various fiber imperfection angle (0.25R) 